\title{
Integration of cardiac magnetic resonance imaging, electrocardiographic imaging, and coronary venous computed tomography angiography for guidance of left ventricular lead positioning
}

Citation for published version (APA):

Nguyen, U. C., Cluitmans, M. J. M., Strik, M., Luermans, J. G., Gommers, S., Wildberger, J. E., Bekkers, S. C. A. M., Volders, P. G. A., Mihl, C., Prinzen, F. W., \& Vernooy, K. (2019). Integration of cardiac magnetic resonance imaging, electrocardiographic imaging, and coronary venous computed tomography angiography for guidance of left ventricular lead positioning. EP Europace, 21(4), 626-635.

https://doi.org/10.1093/europace/euy292

Document status and date:

Published: 01/04/2019

DOI:

10.1093/europace/euy292

Document Version:

Publisher's PDF, also known as Version of record

Document license:

Taverne

Please check the document version of this publication:

- A submitted manuscript is the version of the article upon submission and before peer-review. There can be important differences between the submitted version and the official published version of record. People interested in the research are advised to contact the author for the final version of the publication, or visit the DOI to the publisher's website.

- The final author version and the galley proof are versions of the publication after peer review.

- The final published version features the final layout of the paper including the volume, issue and page numbers.

Link to publication

\footnotetext{
General rights rights.

- You may freely distribute the URL identifying the publication in the public portal. please follow below link for the End User Agreement:

www.umlib.nl/taverne-license

Take down policy

If you believe that this document breaches copyright please contact us at:

repository@maastrichtuniversity.nl

providing details and we will investigate your claim.
}

Copyright and moral rights for the publications made accessible in the public portal are retained by the authors and/or other copyright owners and it is a condition of accessing publications that users recognise and abide by the legal requirements associated with these

- Users may download and print one copy of any publication from the public portal for the purpose of private study or research.

- You may not further distribute the material or use it for any profit-making activity or commercial gain

If the publication is distributed under the terms of Article 25fa of the Dutch Copyright Act, indicated by the "Taverne" license above,

Download date: 26 Apr. 2023 


\title{
Integration of cardiac magnetic resonance imaging, electrocardiographic imaging, and coronary venous computed tomography angiography for guidance of left ventricular lead positioning
}

\author{
Uyên Châu Nguyên ${ }^{1,2 * \dagger \dagger}$, Matthijs J. M. Cluitmans ${ }^{2 \dagger}$, Marc Strik ${ }^{2}$, Justin G. Luermans ${ }^{2}$, \\ Suzanne Gommers ${ }^{3}$, Joachim E. Wildberger ${ }^{3}$, Sebastiaan C. A. M. Bekkers ${ }^{1,3}$, \\ Paul G. A. Volders' ${ }^{2}$, Casper Mihl ${ }^{3}$, Frits W. Prinzen ${ }^{1}$, and Kevin Vernooy ${ }^{2,4}$
}

${ }^{1}$ Department of Physiology, Cardiovascular Research Institute Maastricht (CARIM), Maastricht University Medical Center, PO Box 616, 6200MD, Maastricht, the Netherlands; ${ }^{2}$ Department of Cardiology, CARIM, Maastricht University Medical Center, Maastricht, the Netherlands; ${ }^{3}$ Department of Radiology, CARIM, Maastricht University Medical Center, Maastricht, the Netherlands; and ${ }^{4}$ Department of Cardiology, Radboud University Medical Center, Nijmegen, the Netherlands

Received 21 August 2018; editorial decision 3 November 2018; accepted 12 November 2018; online publish-ahead-of-print 24 December 2018

Aims

An appropriate left ventricular (LV) lead position is a pre-requisite for response to cardiac resynchronization therapy (CRT) and is highly patient-specific. The purpose of this study was to develop a non-invasive pre-procedural CRT-roadmap to guide LV lead placement to a coronary vein in late-activated myocardium remote from scar.

Methods

and results

Sixteen CRT candidates were prospectively included. Electrocardiographic imaging (ECGI), computed tomography angiography (CTA), and delayed enhancement cardiac magnetic resonance imaging (DE-CMR) were integrated into a 3D cardiac model (CRT-roadmap) using anatomic landmarks from CTA and DE-CMR. Electrocardiographic imaging was performed using 184 electrodes and a CT-based heart-torso geometry. Coronary venous anatomy was visualized using a designated CTA protocol. Focal scar was assessed from DE-CMR. Cardiac resynchronization therapy-roadmaps were constructed for all 16 patients [left bundle branch block: $n=6$; intraventricular conduction disturbance: $n=8$; narrow-QRS (ablate and pace strategy); $n=1$; right bundle branch block: $n=1$ ]. The number of coronary veins ranged between 3 and 4 per patient. The CRT-roadmaps showed no $(n=5), 1(n=6)$, or $2(n=5)$ veins per patient located outside scar in late-activated myocardium [ $\geq 50 \%$ QRS duration (QRSd)]. Final LV lead position was outside scar in late-activated myocardium in 11 out of 14 implanted patients, while a LV lead in scar was unavoidable in the remaining three patients.

Conclusion A non-invasive pre-implantation CRT-roadmap was feasible to develop in a case series by integration of coronary venous anatomy, myocardial-scar localization, and epicardial electrical activation patterns, anticipating on clinically relevant features.

Keywords

Cardiac resynchronization therapy $\bullet$ Left ventricular lead • Image integration • Coronary venous anatomy • Computed tomography angiography • Electrocardiographic imaging • Cardiac magnetic resonance imaging • Myocardial scar - Heart failure

\footnotetext{
* Corresponding author. Tel: +31-43-3881200; fax: +31-43-3884166. E-mail address: u.nguyen@maastrichtuniversity.nl

† The first two authors contributed equally to the study.

Published on behalf of the European Society of Cardiology. All rights reserved. @ The Author(s) 2018. For permissions, please email: journals.permissions@oup.com.
} 


\section{What's new?}

- A non-invasive cardiac resynchronization therapy (CRT) roadmap was developed by integration of cardiac magnetic resonance imaging, electrocardiographic imaging, and computed tomography angiography to guide left ventricular lead placement.

- The advantage of the present CRT-roadmap is that it comprises detailed imaging of the coronary veins with respect to the scar substrate localization as well as high-resolution epicardial electrical activation patterns.

- This CRT-roadmap was implemented in a case series to demonstrate its feasibility in anticipating during implantation by the use of a single non-invasive 3D cardiac model.

\section{Introduction}

Cardiac resynchronization therapy (CRT) is an effective treatment for patients with heart failure (HF), impaired left ventricular (LV) systolic function, and prolonged QRS duration (QRSd). Nevertheless, one-third of eligible patients fail to benefit from CRT. ${ }^{1}$ An appropriate LV lead position is a pre-requisite for response to CRT and is highly patient-specific. There is increasing evidence that scar tissue should be avoided in LV lead placement. Pacing in scar regions can impact CRT by causing regional slowing of conduction. Furthermore, a LV-lead position in scar is associated with a diminished clinical response to $\mathrm{CRT}^{2,3}$ and proarrhythmic events. ${ }^{4}$ To date, delayed-enhancement cardiac magnetic resonance imaging (DE-CMR) remains the clinical gold standard for the assessment of focal scar.

Besides avoiding scar, the region of latest activation on the LV inferolateral wall is preferred given that the rationale of biventricular pacing is to resynchronize the LV. A LV lead position with an electrical delay of $\geq 50 \%$ of QRSd is associated with an improved haemodynamic and long-term clinical response to CRT. ${ }^{5,6}$ As the site of late activation is heterogeneously distributed among individuals, ${ }^{7,8}$ mapping local activation is desirable. ${ }^{9}$ Electrocardiographic imaging (ECGI) can reconstruct the electrical activation of the epicardium non-invasively by using body surface potential measurements (BSPM) and a patient-specific heart-torso geometry. ${ }^{10}$ Electrical dyssynchrony and local electrical delay identified by ECGI is representative of epicardial contact mapping, ${ }^{11}$ and may be combined with other imaging modalities non-invasively to guide LV lead placement.

Although it seems that careful positioning of the LV lead is important, coronary venous anatomy puts major constraints on the available LV pacing sites, due to the high inter-patient variability regarding the trajectory, shape, and number of veins. ${ }^{12}$ The coronary venous tree generally does not cover all segments of the LV, and some veins may be difficult to cannulate with the LV lead because of anatomical limitations. Coronary venous anatomy is conventionally visualized by fluoroscopic angiography, although computed tomography angiography (CTA) may enable three-dimensional (3D) non-invasive visualization, which helps in the pre-procedural anticipation on patient-specific coronary venous anatomy.

The objective of this study was to assess the feasibility of a patienttailored implantation by creating a pre-procedural multimodality
CRT-roadmap by integration of 3D images from focal scar localization on DE-CMR, epicardial activation sequence from ECGI, and coronary venous anatomy from CTA. This CRT-roadmap was used in a case series to guide LV lead placement to a coronary vein in an electrically late-activated region remote from scar.

\section{Methods}

\section{Study population}

Consecutive patients referred for CRT device implantation were prospectively enrolled from April till December 2017 at Maastricht University Medical Centre. Patients were orally approached at the outpatient clinic and received a patient information form. Only patients with contraindications for CTA or CMR (e.g. severe kidney dysfunction, contrast media intolerance, and claustrophobia) and who clearly stated that they were not willing to participate in any clinical study, were not actively approached for participation in the study.

Patients underwent ECGI, CTA, and CMR up to 2 months prior to implantation. An overview of all processes involving the CRT-roadmap construction is provided in Figure 1. The institutional Review Board approved the study protocol, and patients gave written consent for participation in the study.

\section{Cardiac magnetic resonance imaging}

Standard clinical CMR images were acquired prior implantation during multiple breath-holds on a 1.5-T system (Philips Ingenia, Best, the Netherlands). A 2D inversion recovery gradient echo sequence was used for delayed enhancement $10 \mathrm{~min}$ after an intravenous bolus of 0.15 $\mathrm{mmol} / \mathrm{kg}$ gadobutrol (Ultravist, Bayer Healthcare, Berlin, Germany). CMR images were analysed offline using customized software CAAS MRV3.4 (Pie Medical Imaging, Maastricht, The Netherlands). A single observer performed the CMR analysis under supervision of an CMR reader with over 10 years of experience (EACVI level 3). Endocardial and epicardial contours were manually traced in end-diastolic and end-systolic shortaxis cine images to determine LV functional parameters, and in short-axis DE-CMR images to define the myocardium and construct a 3D cardiac model.

In case of a single hyperenhanced region, scarred infarct detection was automatically performed by using the full width at half maximum criteria by indicating a region as scarred tissue. In case of multiple and more heterogeneous hyperenhanced regions infarct detection was performed by manually moving the threshold slider incorporated in CAAS. Myocardium with an intensity above the threshold of the slider was subsequently automatically segmented as infarct. Infarct segmentations using the latter technique were than manually edited when necessary. Traced DE-CMR contours (Figure 1) were converted into 3D surface meshes in STL-formats.

\section{Computed tomography of the heart, thorax, and coronary veins}

Computed tomography angiography images were acquired with a third generation dual-source Somatom Definition Force CT scanner (Siemens Healthineers, Forchheim, Germany). A non-enhanced low-dose thorax CT scan was performed to determine the electrode positions for BSPM, followed by a cardiac contrast enhanced CTA scan for ECGI reconstruction purposes and for coronary venous anatomy. A test bolus was injected at the level of the coronary sinus (CS) or ascending aorta to assess optimal delay for contrast administration. Delay was calculated by time-to-peak +10 or +20 s for the CS or ascending aorta, respectively. ${ }^{13}$ 


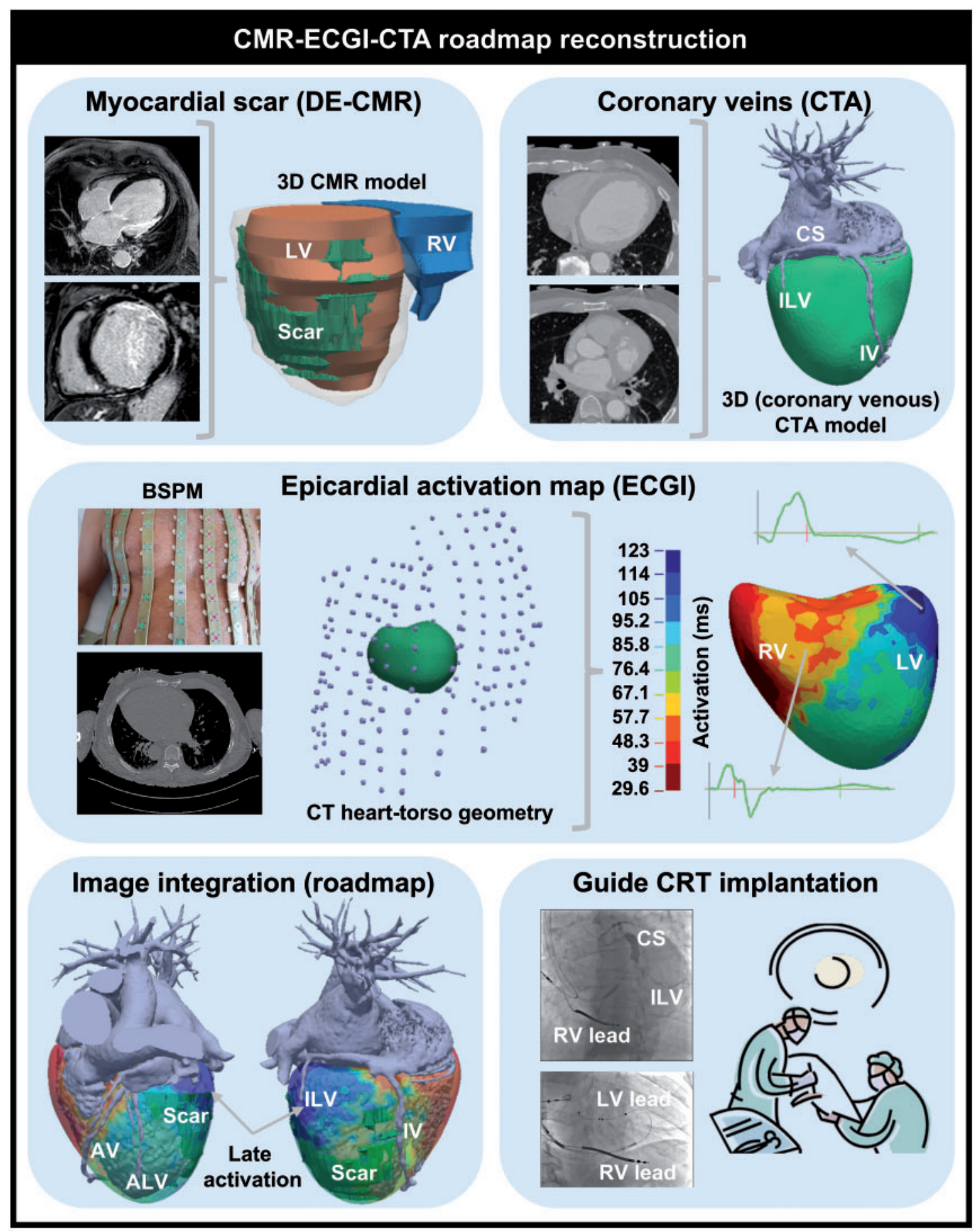

Figure I Workflow for CMR-ECGI-CTA roadmap reconstruction for CRT implantation guidance. Images are taken from Patient 3. AV, anterior vein; BSPM, body surface potential measurement; CMR, cardiac magnetic resonance imaging; CS, coronary sinus; CTA, computed tomography angiography; DE-CMR, delayed enhancement cardiac magnetic resonance imaging; ECGI, electrocardiographic imaging; ILV, inferolateral vein; IV, inferior vein; $L V$, left ventricle; $R V$, right ventricle.

Patients received $72 \mathrm{~mL}$ pre-warmed lopromide 300 (Ultravist, Bayer Healthcare, Berlin, Germany) at a flow rate of $6 \mathrm{~mL} / \mathrm{s}$ directly followed by an $84 \mathrm{~mL}$ mixed bolus (20\% contrast, $80 \%$ saline) at the same flow rate and a $40 \mathrm{~mL}$ saline flush at a flow rate of $3 \mathrm{~mL} / \mathrm{s}$. Total iodine load was $26.6(21.6+5) \mathrm{g}$ iodine. Coronary venous anatomy was classified according to the American Heart Association 17-segment heart model and evaluated under supervision of a radiologist with 7 years' experience in cardiac CTA. Segmentations of the torso, epicardium, blood pool, and coronary veins (Figure 1) were semi-automatically performed in Seg3D v2.4 (SCI, University of Salt Lake City, UT, USA). 


\section{Electrocardiographic imaging: reconstruction and analyses}

Body surface potential measurement recordings of $2 \mathrm{kHz}$ during intrinsic rhythm were carried out with $\sim 184$ torso electrodes using the ActiveTwo system (BioSemi, Amsterdam, the Netherlands). ${ }^{10}$ The epicardial CTA segmentations were re-sampled and smoothed in PreView (SCI, University of Utah, USA) to create a mesh with circa 2000 nodes. All BSPM electrodes were segmented from the low-dose CT thorax in Seg3D and used to build a torso mesh in MATLAB R2016B (MathWorks, Natick, MA, USA). The BSPM recordings and refined epicardium-torso mesh was used for inverse reconstruction (Figure 1) of epicardial potentials using the Tikhonov regularization algorithm. ${ }^{10}$ Epicardial unipolar electrograms (EGM) were reconstructed for every node by concatenating potentials over time. Epicardial activation times were automatically computed from BSPM QRS-onset to the steepest spatiotemporal downslope in the local EGM QRS-complex. ${ }^{10}$

\section{Image integration}

Segmentations of the coronary veins and blood pool from CTA, epicardium from CTA with ECGI reconstructed activation times, and DE-CMR derived endocardium, epicardium, and scar were imported as STL- or VTK-files in the multi-platform data analyses and visualization application software Paraview 5.1.0 (Kitware Inc., Clifton Park, NY, USA). Delayed enhancement cardiac magnetic resonance imaging segmentations were manually translated to match the co-ordinate system of the CTA meshes by creating a (rough) first fit and by fine-tuning from multiple planes. The most important anatomic landmarks used for integration were the LV and RV epicardium, LV endocardium on CMR with the LV blood pool on CTA, interventricular septum, and LV and RV endocardial apex. The appropriateness of the integrated image was based on visual evaluation. After proper translation of the DE-CMR geometry towards the CTA geometry, co-ordinates of the translation matrix ( $\mathrm{X}, \mathrm{Y}$, and Z-direction) were used to visualize the DE-CMR scar substrate on top of the CTA derived ECGI-epicardium, blood pool, and coronary veins. More detailed information on the image integration process is provided in Figure 2. The finalized CRT-roadmap with CMR-ECGI-CTA integration (Figure 1) was presented to the implanting electrophysiologist in $3 \mathrm{D}$ prior and during CRT implantation.

\section{Cardiac resynchronization therapy implantation}

The right atrial lead was targeted towards the right atrial appendage, while the RV lead was targeted towards the apical septum. Left ventricular-lead placement was targeted towards the coronary vein remote from scar in a region of maximum electrical delay on the CRT-roadmap and preferably at the LV free wall. If the major course of the vein from a region with maximal delay on ECGI was located in scar, an alternative vein was targeted remote from scar, provided that the delay was still sufficiently late, preferable $\geq 50 \%$ QRSd. ${ }^{5,6}$

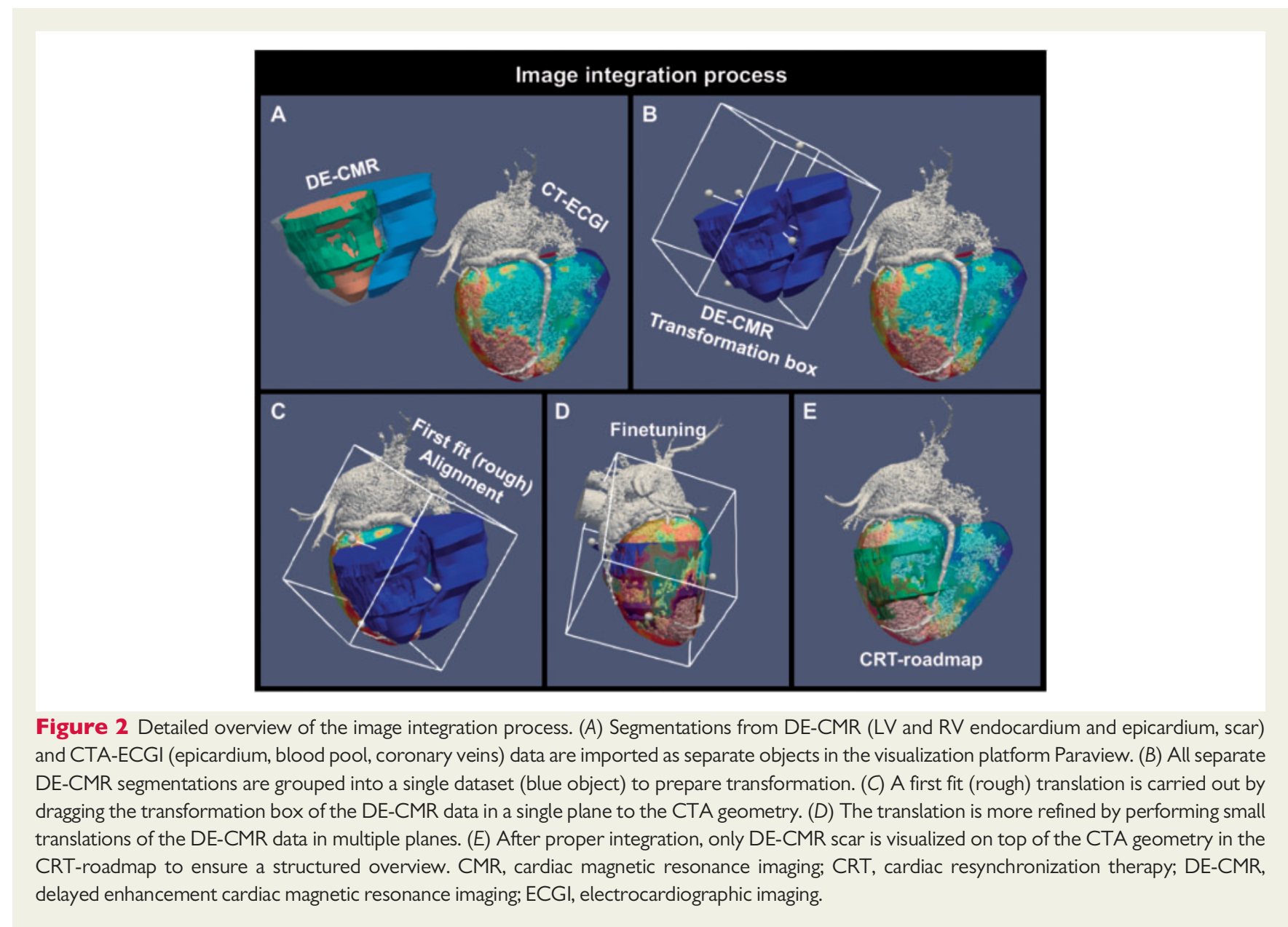


Navigation of the LV lead during the implantation procedure was based on fluoroscopic angiography. Quadripolar LV leads were used as first choice. Several quadripolar leads have been developed with varying shapes and inter-electrode spacing, of which some extend an entire coronary vein. ${ }^{14} \mathrm{~A}$ pacing vector within the target region, low voltage thresholds, without phrenic nerve stimulation, and preferably longest Q-LV was selected for pacing.

To assess the electrical delay at the RV (Q-RV) and LV (Q-LV) lead, simultaneous recording of the surface electrocardiogram (ECG) and sensed RV and LV unipolar EGM during intrinsic rhythm were obtained from the pacemaker/ICD programmer. Q-RV and Q-LV were measured in milliseconds from QRS-onset on the surface ECG to the steepest downslope of the sensed RV or LV EGM.

\section{Data analyses}

Statistical analyses were performed using MATLAB. Continuous variables are expressed as mean \pm standard deviation and dichotomous variables as frequencies and percentages. Final LV lead position in relation to the CRT-roadmap was visually evaluated for every patient. Q-RV and Q-LV measurements were compared with corresponding ECGI delays using Spearman correlation analyses and Wilcoxon signed-rank tests.

\section{Results}

\section{Patient characteristics}

A total of 16 patients planned for CRT device implantation were prospectively included. Patients had a variety of conduction disturbances [left bundle branch block (LBBB): $n=6$, intraventricular conduction disturbance (IVCD): $n=8$; narrow-QRS (ablate and pace strategy): $n=1$; right bundle branch block (RBBB): $n=1$ ]. Fifteen patients were referred for a de novo implantation, and one patient for an upgrade from a dual-chamber pacemaker. Patient characteristics are provided in Table 1.

\section{CMR-electrocardiographic imaging- computed tomography angiography investigations and cardiac resynchronization therapy-roadmap reconstruction}

CMR was performed in 15 out of 16 patients. In one patient who already had a dual-chamber pacemaker, CMR was not performed. The delayed enhancement sequence was of insufficient quality in one patient due to suboptimal cardiac triggering during acquisition. In three patients, DE-CMR was negative, suggesting the absence of scar, while the remaining 11 patients had an average of $20+11 \%$ focal scar in the LV (Table 1).

Coronary venous CTA and low-dose thorax CT was successfully performed in all patients. The number of available coronary veins varied from 3 to 4 per patient. Body surface potential measurements and ECGI reconstructions were also successfully carried out in all patients.

Cardiac resynchronization therapy-roadmaps could be constructed for all 16 patients. In 14 patients, CMR-ECGI-CTA integration was performed; while in two patients only ECGI-CT integrations were created due to absence of adequate DE-CMR data. A representative CRT-roadmap is provided in Figure 3, while a detailed overview of the CMR-ECGI-CTA CRT-roadmaps is provided in Figure 4. There were a total of 57 available coronary veins present on the 16 CRTroadmaps, of which 25 veins were located on the LV lateral wall. Nine out of these 25 LV free wall coronary veins were less suitable for LV-lead placement as these were located in scar or in a region of

Table I Patient characteristics

\begin{tabular}{|c|c|c|c|c|c|c|c|c|c|c|c|}
\hline $\mathbf{P t}$ & Sex & Age & iCMP & NYHA & DE-CMR ${ }^{a}$ & LVEF (\%) & LVEDV (mL) & LVESV (mL) & ECG rhythm & QRS morpholgy & QRSd (ms) \\
\hline 1 & M & 70 & Yes & 2 & $13 \%$ & 28 & 307 & 220 & $\mathrm{AF}$ & IVCD & 134 \\
\hline 2 & M & 67 & Yes & 2 & $21 \%$ & 28 & 278 & 200 & Sinus & LBBB & 152 \\
\hline 3 & M & 72 & Yes & 2 & $15 \%$ & 11 & 505 & 449 & Sinus & LBBB & 138 \\
\hline 4 & $\mathrm{~F}$ & 77 & No & 2 & $0 \%$ & 25 & 232 & 174 & Sinus & LBBB & 148 \\
\hline 5 & $\mathrm{~F}$ & 59 & Yes & 3 & $32 \%$ & 20 & 246 & 197 & Sinus & IVCD & 158 \\
\hline 6 & M & 70 & Yes & 2 & $28 \%$ & 36 & 235 & 150 & Sinus & LBBB & 162 \\
\hline 7 & M & 79 & Yes & 3 & No CMR & 30 & - & - & $\mathrm{RV}$ paced & LBBB & 162 \\
\hline 8 & M & 76 & Yes & 2 & Inadequate & 20 & 359 & 286 & Sinus & IVCD & 150 \\
\hline 9 & $M$ & 61 & No & 3 & $9 \%$ & 43 & 245 & 145 & Sinus & IVCD & 120 \\
\hline 10 & M & 66 & No & 2 & $0 \%$ & 24 & 244 & 186 & Sinus & LBBB & 158 \\
\hline 11 & $M$ & 80 & No & 3 & $31 \%$ & 22 & 275 & 215 & Sinus & IVCD & 122 \\
\hline 12 & $M$ & 76 & Yes & 3 & $10 \%$ & 25 & 266 & 203 & $\mathrm{AF}$ & IVCD & 135 \\
\hline 13 & $\mathrm{~F}$ & 53 & No & 3 & $0 \%$ & 23 & 233 & 161 & Sinus & Narrow & 82 \\
\hline 14 & $M$ & 70 & Yes & 2 & $28 \%$ & 10 & 452 & 413 & Sinus & IVCD & 150 \\
\hline 15 & $M$ & 80 & Yes & 2 & $15 \%$ & 20 & 232 & 181 & $\mathrm{AF}$ & IVCD & 142 \\
\hline 16 & $M$ & 72 & Yes & 2 & $38 \%$ & 23 & 358 & 275 & Sinus & RBBB & 160 \\
\hline
\end{tabular}

AF, atrial fibrillation; DE-CMR, delayed enhancement cardiac magnetic resonance imaging; iCMP, ischemic cardiomyopathy; IVCD, intraventricular conduction disturbance; LBBB, left bundle branch block; LVEDV, left ventricular end-diastolic volume; LVEF, left ventricular ejection fraction; LVESV, left ventricular end-systolic volume; NYHA, New York Heart Association functional class; RBBB, right bundle branch block.

${ }^{a}$ Scar on DE-CMR depicted in \% of LV mass. Left ventricular parameters (LVEF, LVEDV, and LVESV) are all derived from CMR with exception of Patient 7. In Patient 8, the delayed enhancement sequence was inadequate to quantify scar, although cine images were sufficient for evaluation of $L V$ functional parameters. 


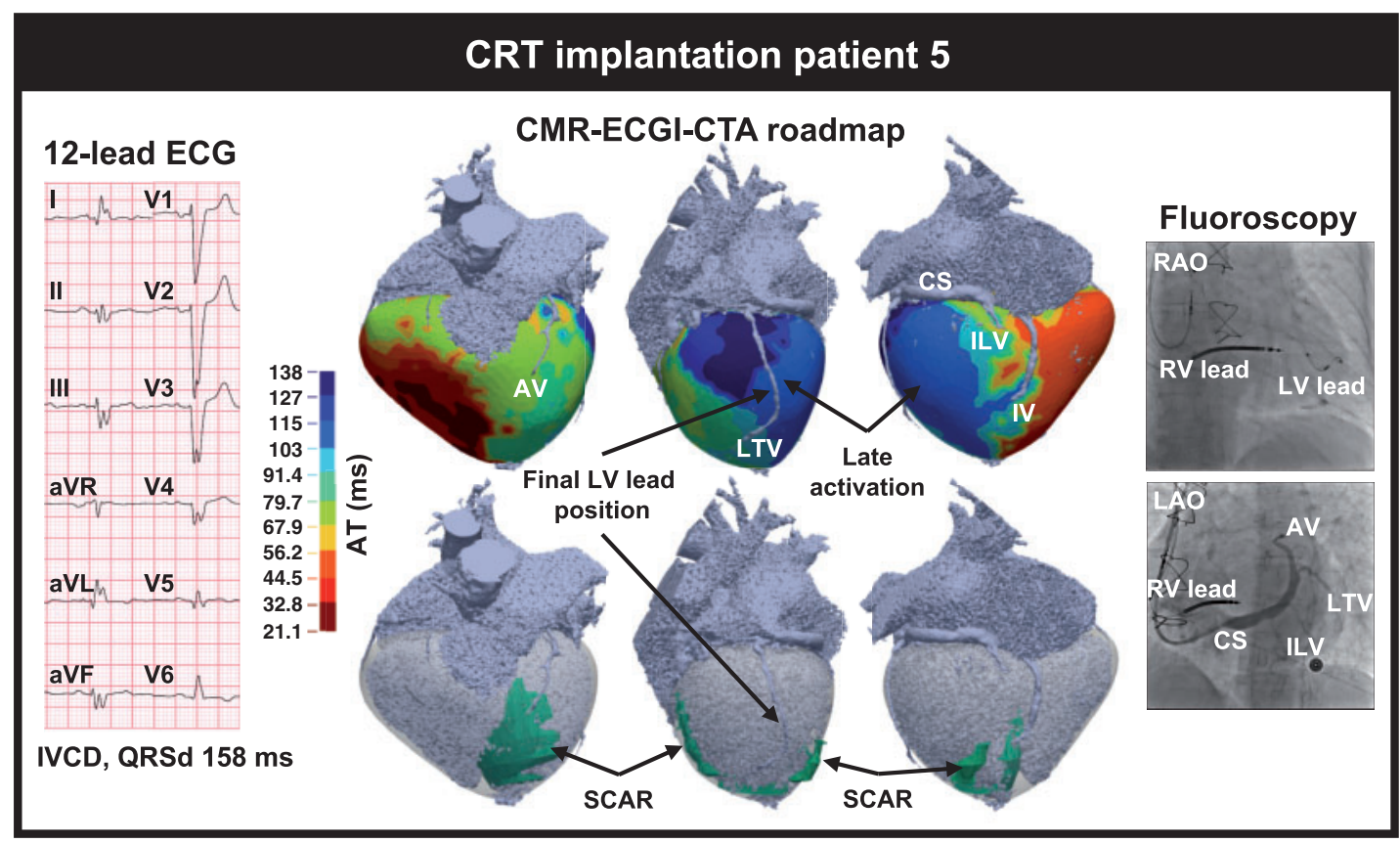

Figure 3 Representative CMR-ECGI-CTA roadmap of Patient 5 for CRT implantation. AT, activation time; AV, anterior vein; CMR, cardiac magnetic resonance imaging; CRT, cardiac resynchronization therapy; CS, coronary sinus; CTA, computed tomography angiography; ECGI, electrocardiographic imaging; ILV, inferolateral vein; IV, inferior vein; IVCD, interventricular conduction disturbance; LAO, left anterior oblique; LTV, lateral vein; $L V$, left ventricle; $R A O$, right anterior oblique.

early activation ( $<50 \%$ QRSd) on the CRT-roadmap. Sixteen out of $25 \mathrm{LV}$ free wall coronary veins were located remote from scar in late-activated myocardium ( $\geq 50 \%$ QRSd) on the CRT-roadmap and subsequently considered suitable for LV-lead placement. The CRTroadmap showed that there were no $(n=5)$, one $(n=6)$, or two $(n=5)$ veins per patient located outside scar in late-activated myocardium ( $\geq 50 \%$ QRSd) on the CRT-roadmap, thus potential candidates for lead positioning.

\section{Cardiac resynchronization therapy device implantation and left ventricular- lead placement}

Fourteen out of 16 patients underwent CRT implantation. In one patient (no. 9) implantation was cancelled after construction of the CRT-roadmap due to improved left ventricular ejection fraction (LVEF) on CMR. One patient (no. 14) died suddenly before the scheduled CRT implantation unrelated to the study protocol or CRT procedure due to an out of hospital cardiac arrest.

A standard left-sided CRT implantation was used in 13 out of 14 patients, while a right-sided implantation approach was carried out in one patient (no. 4) because of a persistent left-sided superior cava with no left innominate vein visualized on the pre-procedural CTA. Mean fluoroscopy time was $32 \pm 9 \mathrm{~min}$, mean radiation dose was $389 \pm 276 \mathrm{mGy}$, and mean total procedural time was $159 \pm 40 \mathrm{~min}$.

Detailed information on the final LV-lead placement in relation to the CRT-roadmap per individual patient is provided in Figure 4 and summarized in Figure 5. The LV lead was positioned outside the scar in 11 out of 14 patients. In the remaining three patients (nos 2, 6, and 16), scar extended to all LV free wall segments and could not be avoided. In two patients (nos 3 and 4) cannulation of the initial target vein was not possible due to limited coronary venous anatomy. Electrocardiographic imaging delay at the LV lead was $\geq 50 \%$ in 13 out of 14 patients and $<50 \%$ in 1 patient. In 11 patients, the LV lead was placed both outside scar and in an area with $\geq 50 \%$ QRSd, and considered as optimal positions.

\section{Electrocardiographic imaging epicardial activation times vs. invasive measurements}

Intra-procedural intrinsic Q-LV and Q-RV measurements were performed in all 14 implanted patients. Three Q-RV measurements were excluded from the analyses, as these patients had a mid-septal $\mathrm{RV}$ lead position as the septum is not reconstructed by ECGI, and therefore the RV lead location was too far located from the ECGI epicardial activation maps. Representative Q-RV and Q-LV measurements are provided in Figure 6. Overall there was a good correlation (R: 0.85, P<0.001) between Q-LV/Q-RV and ECGI delays (Figure 7). Electrocardiographic imaging delays showed lower values compared to invasive measurements (difference $-15 \pm 26 \mathrm{~ms}, P=0.0295$ ).

\section{Discussion}

In the present study, the feasibility of creating a non-invasive pre-implantation CRT-roadmap was evaluated in a case series by integration 


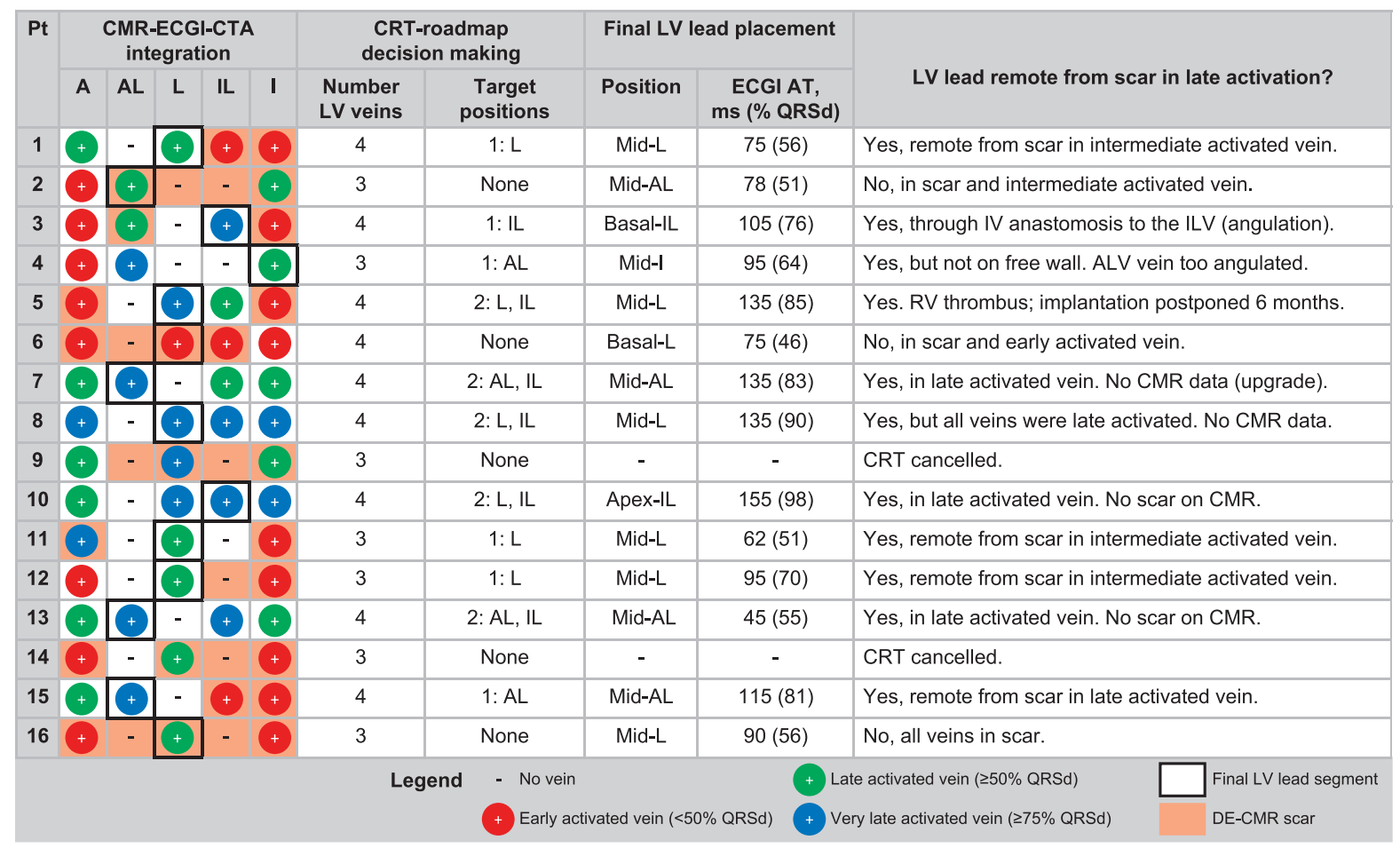

Figure 4 Patient-specific information on the CRT-roadmap and final LV lead placement. CMR-ECGI-CTA integration, CRT-roadmap, and final LV lead position. A, anterior; AL, anterolateral; AT, activation time; CMR, cardiac magnetic resonance imaging; CRT, cardiac resynchronization therapy; CTA, computed tomography angiography; DE-CMR, delayed enhancement cardiac magnetic resonance imaging; ECGI, electrocardiographic imaging; I, inferior; IL, inferolateral; L, lateral; LV, left ventricle; RV, right ventricle.

CMR, ECGI, and CT to guide LV-lead placement. The advantage of the present CRT-roadmap, with respect to all previous imaging-guided approaches, is that it comprises detailed imaging of the coronary veins and scar substrate as well as high-resolution epicardial electrical activation patterns, facilitating decision-making during implantation by the use of a single non-invasive 3D cardiac model.

\section{Towards a tailored left ventricular-lead placement}

Several imaging-guided approaches for LV-lead placement have emerged, of which the largest in this regard are STARTER ${ }^{15}$ and the TARGET $^{2}$ studies. Both studies used echocardiography and demonstrated an association between reduced mortality and a LV lead position in late-activated myocardium. The TARGET study additionally underlined the importance of avoiding scar, by showing an almost five-fold mortality reduction when the LV lead was placed outside scar. $^{2}$ The importance of guiding LV lead placement away from scar using DE-CMR is further emphasized in a large cohort of consecutive CRT candidates by Leyva et al. ${ }^{3}$ A LV lead position in scar compared to non-scar was associated with an over 6-fold increase in cardiac death and an increased risk for hospitalization and major adverse cardiovascular events. ${ }^{3}$

A recent smaller study by Kockova et al. ${ }^{16}$ compared an CMRguided (away from scar, in late mechanical activation) LV lead placement approach with a Q-LV guided approach. Interestingly, the use of CMR did not lead to a significant reduction in cardiovascular death or hospitalization, suggestion that the use of Q-LV solely may diminish the relative benefit from CMR. ${ }^{16}$

More refined approaches incorporating multimodality imaging to tailor LV-lead placement have also emerged. Pre-procedural 2D bullseye plots with information on CT, DE-CMR, and echocardiography were constructed by Bakos et al. to define a late-activated segment outside scar. Interestingly, in about half of the patients, the target segment couldn't be reached due to lack of coronary veins, confirming in the importance of visualizing the coronary venous anatomy. $^{17}$

Recently, Behar et al. overlaid CMR-derived scar and mechanical activation on the fluoroscopic angiogram to guide LV-lead placement. ${ }^{18}$ The benefit of the latter approach was that the CMR scanner was installed in the same facility as the catheter laboratory enabling real-time imaging and CRT implantation in a single procedure, enhancing clinical workflow.

The majority of all imaging-guided CRT studies incorporate mechanical activation. As CRT is primarily an electrical therapy, a more detailed assessment of local electrical activation may provide incremental value., ${ }^{5,6}$ In the present study local epicardial electrical activation was assessed non-invasively, using an in-house developed ECGI system validated in animal models. ${ }^{10}$ A good correlation was additionally found between invasive electrical delay measurements and ECGI activation times. 


\section{Final LV lead position}

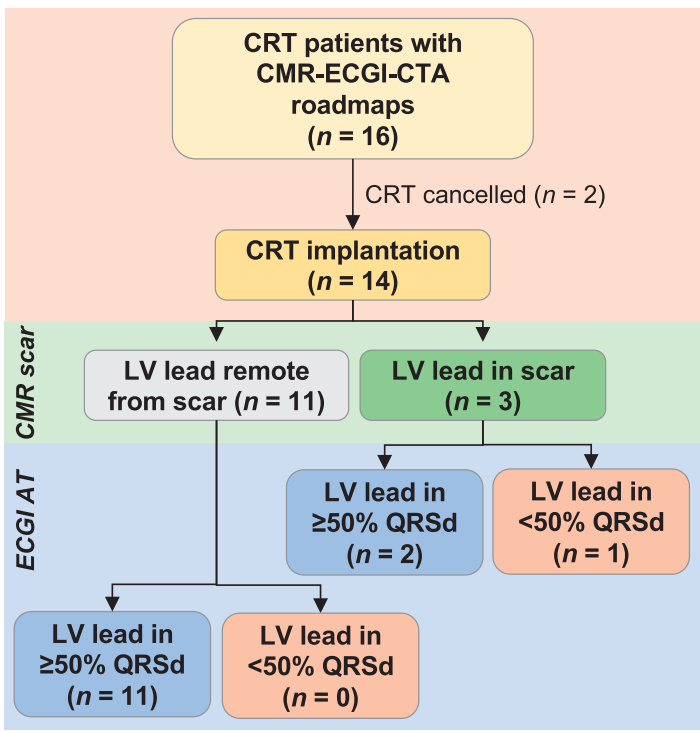

Figure 5 Final LV lead position flowchart. AT, activation time; CMR, cardiac magnetic resonance imaging; CRT, cardiac resynchronization therapy; CTA, computed tomography angiography; ECGI, electrocardiographic imaging; LV, left ventricle.

Only a few other studies incorporated electrical activation for LV lead guidance. Invasive coronary venous electro-anatomic mapping ${ }^{7}$ has been used by our group and has been integrated with DE-CMR ${ }^{9}$ to navigate LV-lead placement intra-procedurally. Although a LV lead position remote from scar in late electrical activation was achieved in the majority of patients, its application requires refined skills of the implanting physician to map all coronary veins.

One study by Dawoud et al. ${ }^{19}$ similarly incorporated coronary venous anatomy, scar extent, and electrical activation non-invasively for CRT by integration of CT with ECGI. The authors subsequently demonstrated the feasibility of ECGI-CT integration in a pre-clinical setting of 6 canine models.

The present CRT-roadmap requires both $C T$ and CMR imaging and thus may be more complex than the use of solely $\mathrm{CMR}^{18}$ or echocardiography. 2,15

\section{Clinical implications}

In the present study, the LV lead was properly positioned remote from scar tissue and in late-activated myocardium in $79 \%$ of patients. The CRT-roadmap in the current study was primarily used for LV-lead placement, but its application in patient selection by computing interventricular dyssynchrony ${ }^{11,20}$ from ECGI together with information on scar and coronary venous anatomy is well within range and an important goal in the near future. Electrocardiographic imaging parameters reflecting interventricular dyssynchrony superior in CRT response prediction compared to the conventional 12-lead ECG include ventricular electrical uncoupling ${ }^{20}$ and activation delay vector. ${ }^{21}$

Visualization of the coronary venous anatomy by CTA preprocedurally may support planning of the implantation strategy. Additionally, using the current CTA protocol yielded a high concordance (85\%) between coronary venous CTA and fluoroscopic angiography. ${ }^{13}$ In the current study, cannulation of the initial target vein was not possible due to suboptimal coronary venous anatomy (angulation at the ostium in Patients 3 and 4 described in Figure 4) and in one patient, a right-sided implantation approach was used due to a persistent left-sided superior vena cava (Patient 5). In case of suboptimal coronary venous anatomy or extensive scarring around the veins, one could anticipate by the use of specific lead types or even consider alternative approaches for biventricular pacing including a surgical placed epicardial lead, endocardial pacing, ${ }^{22}$ or transseptal pacing. ${ }^{23}$

The issue of radiation exposure from CTA however merits discussion. The radiation dose of a standard CTA acquired using an advanced multidector computed tomography scanner is associated with an effective dose of $0.21 \mathrm{mSv} .{ }^{24}$ Despite the radiation burden, CTA had several benefits including a high spatial resolution which is particularly relevant for visualization of small and delicate blood vessels, ${ }^{25}$ and a short acquisition time making it a patient friendly technique. Furthermore, the quantity of radiation exposure may be kept at a minimum by advancing the acquisition protocol. ${ }^{26}$ The amount of radiation can also be put into perspective as it is only a small fraction compared to the amount of radiation exposed to the patient during CRT implantation. The entrance skin dose during CRT implantation may depend on the implanting physician and complexity of the procedure (e.g. fluoroscopy and implantation time), though doses of $1000 \pm 600$ mGy have been reported. ${ }^{27}$

Electrocardiographic imaging alone could be of added value post implantation as well, by assessing the levels of dyssynchrony during different pace settings, potentially improving CRT delivery. With our current ECGI system, extensive ECGI measurements post implantation, can reliably be performed using the original heart-torso geometry exempting the need for a repeat $\mathrm{CT}{ }^{28}$

The utility of CMR-ECGI-CTA integration may even stretch beyond that of CRT alone. A 3D cardiac CMR-ECGI-CTA model could, for instance, be used to localize the origin of premature ventricular contractions or ventricular tachycardia (VT) and guide ablation whilst still avoiding injury of vulnerable coronary arteries or nerves. In a recent study by Cuculich et al., ${ }^{29}$ ECGI was integrated with coronary arteries on CT and scar or ischemia on CMR or single photon emission computed tomography to guide VT ablation, pioneering a complete noninvasive approach of diagnostics and therapy in electrophysiology.

\section{Limitations}

This was a case series study with a limited number of patients. Additionally, baseline functional parameters including LVEF and volumes were based on CMR measurements, prohibiting follow-up measurements by the same imaging technique as CMR may be contraindicated in patients with some CRT device types. Nowadays, United Stated Food and Drug administration approved CMR compatible CRT devices have emerged, ${ }^{30}$ enabling the use of CMR for outcome assessment for instance by assessing LV volumes and mechanical changes (e.g. feature tracking from cine CMR of tagging) after CRT implantation. Although these some CRT devices may be CMR compatible, the device can still cause substantial artefacts, potentially affecting the reliability of $L V$ volume assessments.

The scope of this study was to explore the technical feasibility of the CRT-roadmap. The superiority of such image-guided approach compared to conventional implantation strategies requires a clinical 


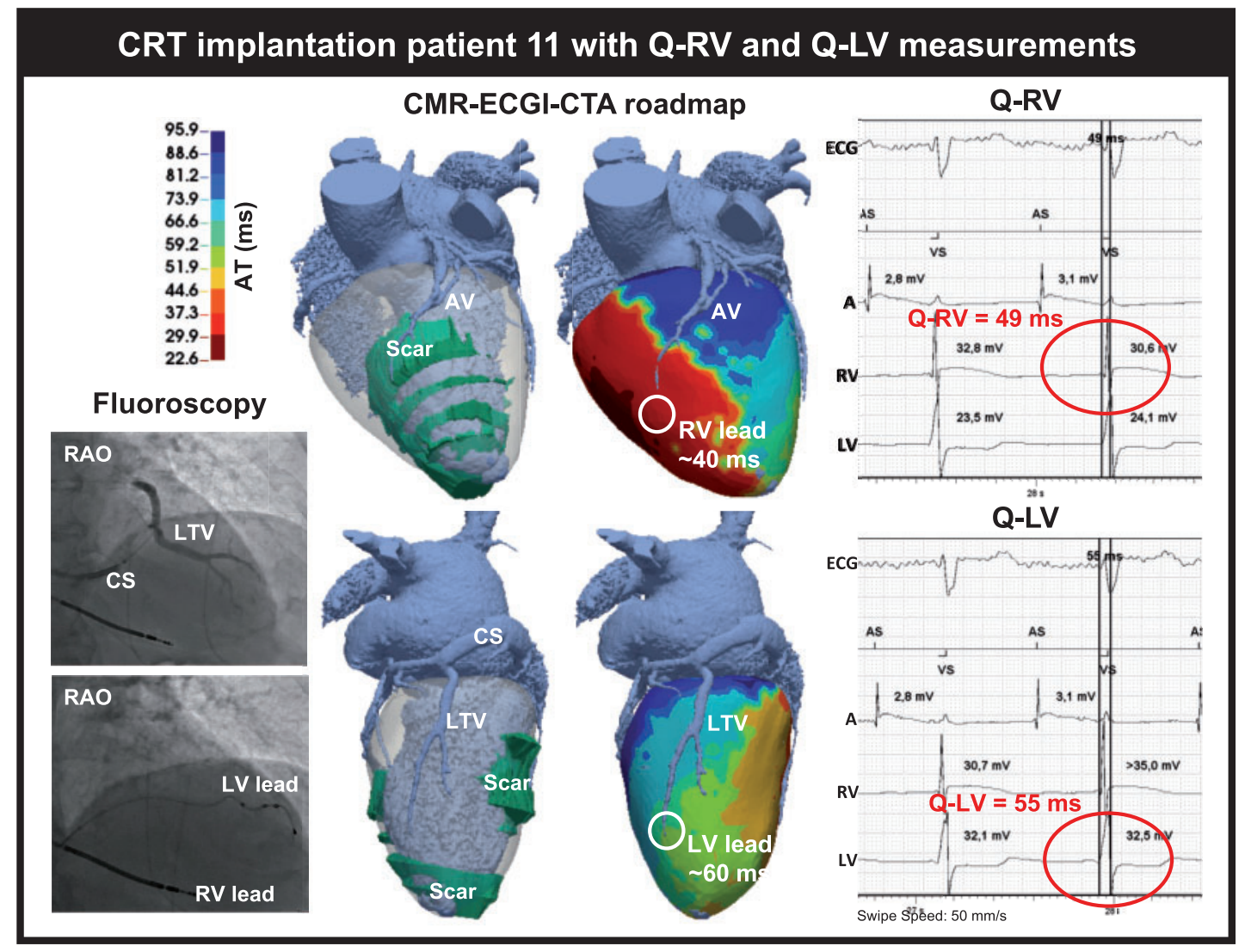

Figure 6 Electrocardiographic imaging activation map of Patient 11 with invasive Q-RV and Q-LV measurements. AT, activation time; AV, anterior vein; CMR, cardiac magnetic resonance imaging; CRT, cardiac resynchronization therapy; CS, coronary sinus; CTA, computed tomography angiography; ECGI, electrocardiographic imaging; LAO, left anterior oblique; LTV, lateral vein; LV, left ventricle; RAO, right anterior oblique; RV, right ventricle.

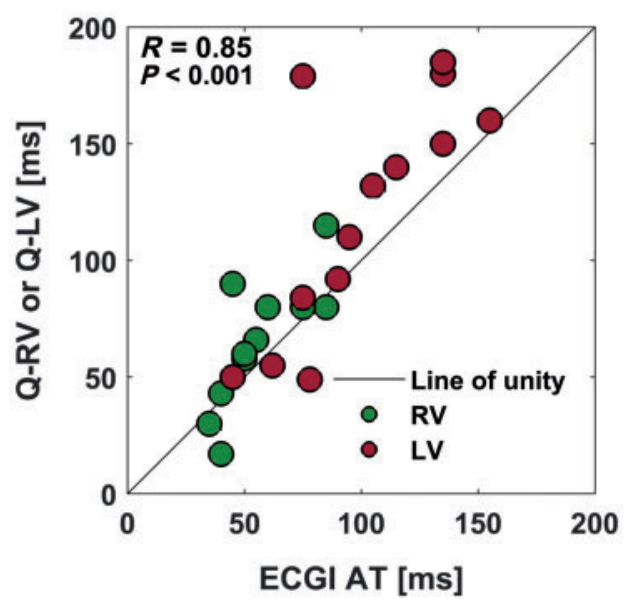

Figure 7 Spearman correlation analysis between activation time (AT) measured intra-procedurally at the RV and LV lead (Q-RV and Q-LV, respectively) vs. associated ECGI AT. ECGI, electrocardiographic imaging; LV, left ventricle; RV, right ventricle. trial with LV reverse remodelling as primary endpoints, randomizing an image-guided approach, using the CMR-ECGI-CTA roadmap, vs. a conventional LV lead placement. Secondary endpoints may include New York Heart Association functional class improvement, mortality, hospitalization, while tertiary endpoints may include ECG characteristics, procedural times, and radiation burden.

Potential differences between coronary venous anatomy imaged by CTA and fluoroscopic angiography may exist due to different stages of congestion and the time difference between the two investigations. All of our patients were clinical stable with respect to their HF status and did not suffer from acute decompensation in the time interval between the CTA exam and CRT implantation.

CMR can be contra-indicated in patients with existing pacing systems. In this subgroup of CRT candidates, alternative CT metrics for scar such as wall thinning, wall motion, ${ }^{12}$ or delayed enhancement CT could be considered.

\section{Conclusion}

In the present study, the development and implementation of a novel CRT-roadmap, that comprehends detailed imaging of the coronary 
veins and scar substrate as well as high-resolution epicardial electrical activation patterns, was evaluated in a case series. The feasibility of this CRT-roadmap in facilitating decision-making during implantation by the use of a single non-invasive 3D cardiac model is demonstrated, potentially improving outcome.

\section{Funding}

Kootstra Talent Fellowship research grant from Maastricht University to U.C.N.; and Dutch Heart Foundation (grant \#2015T61 to M.S.).

Conflict of interest: M.C. is supported by the Hein Wellens Fonds and is part-time employed by Philips. F.W.P. has received research grants from Medtronic, Abbott Medical, LivaNova, Biosense Webster, MSD, and Biotronik. KV received research grants from Medtronic. J.E.W. has received research grants from AGFA, Bard, Bayer, GE, Optimed, Philips, Siemens and speaker fees from Bayer, Siemens. The authors declare that no competing interests exist.

\section{References}

1. Auricchio A, Prinzen FW. Non-responders to cardiac resynchronization therapy: the magnitude of the problem and the issues. Circ J 2011;75:521-7.

2. Khan FZ, Virdee MS, Palmer CR, Pugh PJ, O'Halloran D, Elsik M et al. Targeted left ventricular lead placement to guide cardiac resynchronization therapy: the TARGET study: a randomized, controlled trial. J Am Coll Cardiol 2012;59: 1509-18.

3. Leyva F, Foley PW, Chalil S, Ratib K, Smith RE, Prinzen F et al. Cardiac resynchronization therapy guided by late gadolinium-enhancement cardiovascular magnetic resonance. J Cardiovasc Magn Reson 2011;13:29.

4. Roque C, Trevisi N, Silberbauer J, Oloriz T, Mizuno H, Baratto F et al. Electrical storm induced by cardiac resynchronization therapy is determined by pacing on epicardial scar and can be successfully managed by catheter ablation. Circ Arrhythm Electrophysiol 2014;7:1064-9.

5. Singh JP, Fan D, Heist EK, Alabiad CR, Taub C, Reddy V et al. Left ventricular lead electrical delay predicts response to cardiac resynchronization therapy. Heart Rhythm 2006;3:1285-92.

6. Kandala J, Upadhyay GA, Altman RK, Parks KA, Orencole M, Mela T et al. QRS morphology, left ventricular lead location, and clinical outcome in patients receiving cardiac resynchronization therapy. Eur Heart J 2013;34:2252-62.

7. Rad MM, Blaauw Y, Dinh T, Pison L, Crijns HJ, Prinzen FW et al. Left ventricular lead placement in the latest activated region guided by coronary venous electroanatomic mapping. Europace 2015;17:84-93.

8. Rudy Y. Noninvasive electrocardiographic imaging of cardiac resynchronization therapy in patients with heart failure. J Electrocardiol 2006;39(Suppl 4):S28-30.

9. Nguyen UC, Mafi-Rad M, Aben JP, Smulders MW, Engels EB, van Stipdonk AM et al. A novel approach for left ventricular lead placement in cardiac resynchronization therapy: intraprocedural integration of coronary venous electroanatomic mapping with delayed enhancement cardiac magnetic resonance imaging. Heart Rhythm 2017;14:110-9.

10. Cluitmans MJM, Bonizzi P, Karel JMH, Das M, Kietselaer BLJH, de Jong MMJ et al. In vivo validation of electrocardiographic imaging. JACC: Clin Electrophysiol 2017;3: 232-42.

11. Bear LR, Huntjens PR, Walton RD, Bernus O, Coronel R, Dubois R. Cardiac electrical dyssynchrony is accurately detected by noninvasive electrocardiographic imaging. Heart Rhythm 2018;15:1058-69.

12. Truong QA, Szymonifka J, Picard MH, Thai WE, Wai B, Cheung JW et al. Utility of dual-source computed tomography in cardiac resynchronization therapyDIRECT study. Heart Rhythm 2018;15:1206-13.
13. Nguyên UC, Cluitmans MJM, Luermans JGLM, Strik M, de Vos CB, Kietselaer $\mathrm{BLJH}$ et al. Visualisation of coronary venous anatomy by computed tomography angiography prior to cardiac resynchronisation therapy implantation. Neth Heart | 2018;26:433-44

14. van Everdingen WM, Cramer MJ, Doevendans PA, Meine M. Quadripolar leads in cardiac resynchronization therapy. JACC Clin Electrophysiol 2015;1:225-37.

15. Saba S, Marek J, Schwartzman D, Jain S, Adelstein E, White $P$ et al. Echocardiography-guided left ventricular lead placement for cardiac resynchronization therapy: results of the Speckle Tracking Assisted Resynchronization Therapy for Electrode Region trial. Circ Heart Fail 2013;6:427-34.

16. Kockova R, Sedlacek K, Wichterle D, Sikula V, Tintera J, Jansova H et al. Cardiac resynchronization therapy guided by cardiac magnetic resonance imaging: a prospective, single-centre randomized study (CMR-CRT). Int J Cardiol 2018;270: 325-30.

17. Bakos Z, Markstad H, Ostenfeld E, Carlsson M, Roijer A, Borgquist R. Combined preoperative information using a bullseye plot from speckle tracking echocardiography, cardiac CT scan, and MRI scan: targeted left ventricular lead implantation in patients receiving cardiac resynchronization therapy. Eur Heart Cardiovasc Imaging 2014;15:523-31.

18. Behar JM, Mountney P, Toth D, Reiml S, Panayiotou M, Brost A et al. Real-time X-MRI-guided left ventricular lead implantation for targeted delivery of cardiac resynchronization therapy. JACC: Clin Electrophysiol 2017;3:803-14.

19. Dawoud F, Schuleri K, Spragg D, Horáček B, Berger R, Halperin H et al. Insights from novel noninvasive CT and ECG imaging modalities on electromechanical myocardial activation in a canine model of ischemic dyssynchronous heart failure. J Cardiovasc Electrophysiol 2016;27:1454-61.

20. Ploux S, Lumens J, Whinnett Z, Montaudon M, Strom M, Ramanathan C et al. Noninvasive electrocardiographic mapping to improve patient selection for cardiac resynchronization therapy: beyond QRS duration and left bundle branch block morphology. J Am Coll Cardiol 2013;61:2435-43.

21. Strik M, Ploux S, Huntjens PR, Nguyen UC, Frontera A, Eschalier R et al. Response to cardiac resynchronization therapy is determined by intrinsic electrical substrate rather than by its modification. Int J Cardiol 2018;270:143-8.

22. Strik M, Rademakers LM, van Deursen C], van Hunnik A, Kuiper M, Klersy C et al. Endocardial left ventricular pacing improves cardiac resynchronization therapy in chronic asynchronous infarction and heart failure models. Circ Arrhythm Electrophysiol 2012;5:191-200.

23. Mafi-Rad M, Luermans JG, Blaauw Y, Janssen M, Crijns HJ, Prinzen FW et al. Feasibility and acute hemodynamic effect of left ventricular septal pacing by transvenous approach through the interventricular septum. Circ Arrhythm Electrophysiol 2016;9:e003344.

24. Benz DC, Fuchs TA, Grani C, Studer Bruengger AA, Clerc OF, Mikulicic F et al. Head-to-head comparison of adaptive statistical and model-based iterative reconstruction algorithms for submillisievert coronary CT angiography. Eur Heart Cardiovasc Imaging 2018;19:193-8.

25. Chen YA, Nguyen ET, Dennie C, Wald RM, Crean AM, Yoo SJ et al. Computed tomography and magnetic resonance imaging of the coronary sinus: anatomic variants and congenital anomalies. Insights Imaging 2014;5:547-57.

26. Faure ME, Swart LE, Dijkshoorn ML, Bekkers JA, van Straten M, Nieman K et al. Advanced CT acquisition protocol with a third-generation dual-source CT scanner and iterative reconstruction technique for comprehensive prosthetic heart valve assessment. Eur Radiol 2018;28:2159-68.

27. Suzuki S, Furui S, Yamakawa T, Isshiki T, Watanabe A, lino R et al. Radiation exposure to patients' skin during cardiac resynchronization therapy. Europace 2009; 11:1683-8.

28. Cluitmans MJM, Nguyen UC, Volders P. Follow-up electrocardiographic imaging does not require repeat imaging of body-surface electrodes. Europace 2018 20(Suppl 1):i234.

29. Cuculich PS, Schill MR, Kashani R, Mutic S, Lang A, Cooper D et al. Noninvasive cardiac radiation for ablation of ventricular tachycardia. N Engl J Med 2017;377: 2325-36.

30. Muthalaly RG, Nerlekar N, Ge Y, Kwong RY, Nasis A. MRI in patients with cardiac implantable electronic devices. Radiology 2018;289:281-92. 